\title{
Two teenagers, traumatic aortic disruption, and transoesophageal echocardiography
}

\author{
Anthony Absalom, Alys Burns, Francis Wells, David Stone
}

\begin{abstract}
A report of two teenagers is presented who were passengers of the same vehicle when it was involved in a serious road traffic accident. Their vehicle was hit on the side at high speed by a second vehicle. Both teenagers sustained multiple injuries. On arrival at a district general hospital they required resuscitation, exploratory laparotomies, and postoperative intensive care. Neither had radiographic evidence to suggest any mediastinal injury, but transoesophageal echocardiography demonstrated aortic disruption in both patients. They were transferred to a regional cardiothoracic centre where their lesions were successfully repaired and both have made a full recovery.
\end{abstract}

(Heart 1997;78:310-312)

Keywords: traumatic aortic disruption; transoesophageal echocardiography; district general hospital

Aortic disruption is one of several serious injuries that may result from blunt chest trauma. The lethal nature of the injury has been recognised for a long time and was emphasised by Strassman in $1947 .{ }^{1}$ The risk of death is greatest either on impact or immediately after, and only $20 \%$ of patients will reach hospital alive. ${ }^{2}$ As time passes the risk of sudden death decreases but the risk of sudden exsanguinating haemorrhage remains. ${ }^{3}$ Even if they survive for 10 years after the trauma $20 \%$ of patients with a residual false aneurysm will die of rupture in the subsequent five years. ${ }^{45}$

Prompt diagnosis and treatment are essential. With appropriate management the survival rate of those that reach hospital alive approaches $80 \%,{ }^{6}$ otherwise the six week mortality is $90 \% .^{3}$ Intrathoracic injuries should be excluded in all patients who have been involved in accidents involving blunt chest trauma as well as those with radiographic signs of mediastinal haematoma or other radiographic signs indicating significant kinetic energy transmission. A high index of suspicion for these injuries is vital as they may be present with no external physical signs of aortic injury ${ }^{6}$ and the chest radiograph can be normal. ${ }^{7}$
We report the case of two teenagers who were involved in a road traffic accident and who both sustained an aortic tear, diagnosed by transoesophageal echocardiography at the district general hospital where they were treated initially.

\section{Case report}

Four teenagers were occupants of a small car when it was struck on the left hand side as it turned into the path of an oncoming vehicle. Their vehicle was travelling slowly whereas the oncoming vehicle was travelling at high speed. The two occupants who had sat on the side of the impact (the subjects of this case report) were trapped in the wreckage and sustained multiple injuries; the other occupants of the car and the driver of the second vehicle sustained only minor injuries.

\section{CASE 1}

The seriously injured passenger in the front of the vehicle was a 17 year old woman who had been wearing a seat belt. On arrival at our accident unit she had a Glasgow Coma Score of $7 / 15$ and was haemodynamically unstable. She required fluid resuscitation, and urgent intubation and ventilation because of head injury and respiratory distress. She had multiple, bilateral rib fractures with a flail chest, and a haemopneumothorax on the right. Cranial computed tomography was normal. Her abdomen was distended and tender, and at laparotomy a ruptured spleen was removed. Other injuries included scalp and facial lacerations, and fractures of her nasal bones, mandible, pelvis, and left olecranon process.

She was ventilated postoperatively in the intensive care unit where she remained stable. However, a systolic murmur was noted in the right second intercostal space, radiating up both carotid arteries. Her electrocardiograph was normal and a supine anteroposterior chest radiograph showed no evidence of mediastinal haematoma. Transthoracic echocardiography was unhelpful because of mediastinal shift to the right, therefore, transoesophageal echocardiography was performed (Hewlett-Packard Sonos 1000 ultrasound transoesophageal $5 \mathrm{MHz}$ biplane transducer probe). This showed a suspicious lesion in the initial part of the descending aorta (fig 1). The patient was 




Figure 1 Transoesophageal echocardiogram (longitudinal view) of the descending aorta in case 1 showing lesion suggesting an intimal flap.

transferred to a regional cardiothoracic centre where an aortogram performed on arrival demonstrated an aortic transection.

She was immediately transferred to the operating theatre where a left posterolateral thoracotomy through the fourth intercostal space was performed. Approximately $500 \mathrm{ml}$ of blood was found in the pleural space, with a large bruise over the proximal descending aorta. Clamps were applied to the aortic arch proximally between the left common carotid and subclavian arteries, and distally about $4 \mathrm{~cm}$ below the haematoma. There was an aortic transection just distal to the ligamentum arteriosum. This was exposed by incising the adventitia and cutting back to fresh tissue. The ends were joined directly with a running 3/0 prolene suture. Cross-clamp time was $10 \mathrm{~min}$ utes obviating the need for any bypass support or shunt.

She made a good recovery, although initially she required a tracheostomy to aid weaning from mechanical ventilation. She was discharged home three weeks after the accident.

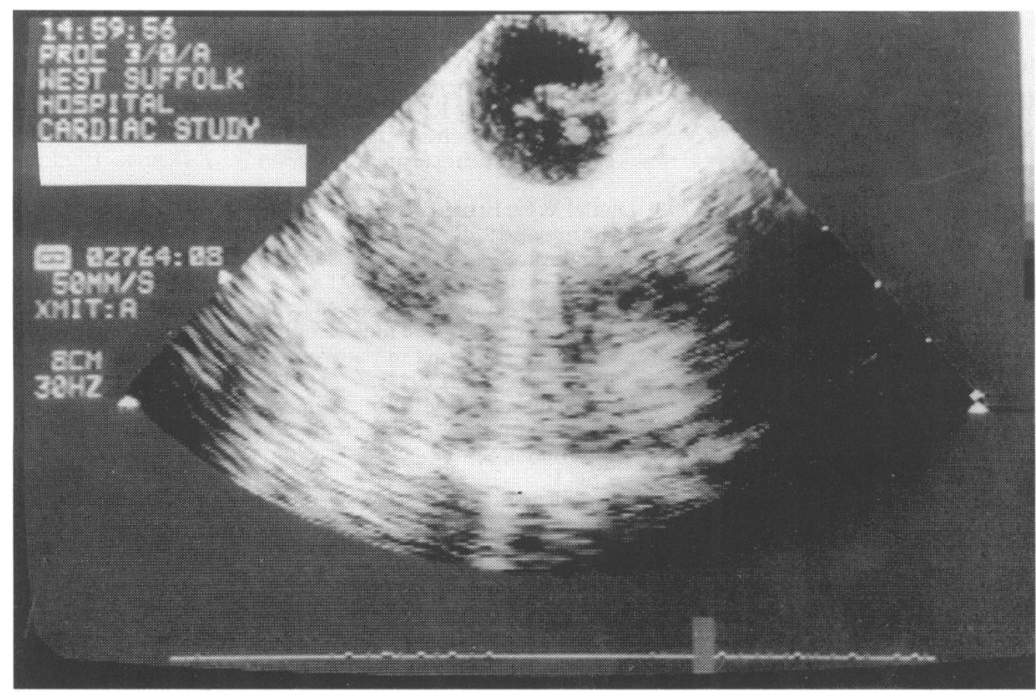

Figure 2 Transoesophageal echocardiogram (cross sectional view) of descending aorta showing aortic disruption in case 2.
CASE 2

A 17 year old man had been sitting on the left hand side of the rear seat, unrestrained. He sustained pulmonary contusions, a ruptured diaphragm, fractured ribs (left side, ribs 2-6), and a splenic capsular tear. Despite these injuries he remained haemodynamically stable and did not require ventilation before laparotomy and thoracotomy, during which his diaphragm was repaired. His splenic tear was treated conservatively.

Over the following 36 hours he made a good recovery from the trauma of the accident and subsequent surgery. There were no signs, clinically or on supine anteroposterior chest radiographs, to suggest aortic injury. On the second postoperative day he was ready for extubation but, knowing that the impact he had suffered was similar to that suffered by the female passenger we decided to perform transoesophageal echocardiography. This showed an abnormal aortic arch with an appearance suggesting an intimal flap (fig 2). $\mathrm{He}$ was transferred to the same regional centre as the female patient where aortography demonstrated an aortic transection just distal to the origin of the left subclavian artery with an annular bulge just cranial to the transection.

Surgical repair proceeded in a similar fashion to that in case 1 , although some variations in technique were required. The ligamentum arteriosum had to be ligated and divided to allow full mobilisation of the aorta. After the occluding clamps were applied there was significant backflow from the distal aorta which obscured the surgical field. This was from large intercostal arteries and was solved by first anastomosing a precoated Dacron graft to the distal end and then applying a cross-clamp to the graft while the proximal anastomosis was completed. The cross-clamp time was 30 minutes and no shunt or bypass was used. The patient was discharged home one week later.

\section{Discussion}

To our knowledge this is the first published report of two cases of traumatic aortic transection from one motor vehicle. We feel this case highlights several important issues.

Knowledge of the mechanism of injury will provide important clues to the possibility of an intrathoracic injury. Patients sustaining acceleration-deceleration injuries are particularly at risk for mediastinal injuries. When an impact causes displacement of the heart and major vessels the portions of the aorta fixed by surrounding structures undergo a high degree of stress, which is why the most common sites of traumatic aortic rupture are the proximal descending aorta in the region of the ligamentum arteriosum, the distal descending thoracic aorta at the level of the diaphragm, and the ascending aorta at the sinuses of Valsalva.

Another issue is the lack of sensitivity and specificity of the chest radiograph in the diagnosis of aortic rupture. Standard chest $x$ rays can provide clues, but the radiograph can be normal in up to $20 \%$ of patients with an aortic injury. ${ }^{8}$ Of patients with evidence on radiogra- 
phy of a wide mediastinum only $10 \%$ will have an aortic injury demonstrated on aortography. ${ }^{9}$ More sensitive and specific procedures are required for confirmation of the diagnosis.? While our patients had radiographic evidence of significant kinetic energy transfer (high rib fractures and a scapular fracture) neither had evidence of a mediastinal haematoma.

A third issue is the choice of diagnostic method used in the evaluation of a patient with a suspected aortic injury. Aortography has long been regarded as the gold standard, but recent papers have reported that transoesophageal echocardiography compares favourably with aortography and some have even recommended the former as the primary imaging technique in patients at risk of aortic injury. ${ }^{7}$ Karalis et al found that transoesophageal echocardiography was more sensitive than aortography at detecting small intimal tears of the descending thoracic aorta. ${ }^{10}$ Transoesophageal echocardiography is much quicker to perform than aortography (24 $v 75$ minutes in a recent study) ${ }^{7}$ and has fewer risks. Daniel et al reported on a multicentre survey of 10419 transoesophageal echocardiography examinations and found a complication rate of only $0.88 \%{ }^{11}$ whereas the incidence of complications following aortography is $1-10 \% .{ }^{12}$ Transoesophageal echocardiography does have some disadvantages including a limited view of the distal ascending aorta and proximal aortic arch owing to the interposition of the trachea between the aorta and oesophagus at this level. Alternative investigations to transoesophageal echocardiography and aortography are contrast enhanced computed tomography, magnetic resonance imaging, ultrafast and spiral computed tomography, and transthoracic echocardiography.

Many district general hospitals have neither transoesophageal echocardiography nor aortography facilities. Computed tomography is usually available but its role is controversial and some regard it as having no part in the investigation of patients at risk of traumatic aortic injury. ${ }^{13}$ Most peripheral hospitals do have transthoracic echocardiography, but it is of limited value in patients with blunt chest trauma (13-28\% will have suboptimal examinations ${ }^{10}$ ). A negative finding on transthoracic echocardiography does not exclude an aortic injury as its sensitivity is only about $60 \%{ }^{14}$ The retrocardiac position of the oesophagus confers several benefits which make transoesophageal superior to transthoracic echocardiography. This approach allows better visualisation of the heart, ascending and descending aorta, and, as no bone penetration is required, higher frequencies may be used, which yield much higher resolution images.

The role of transoesophageal echocardiography in the peripheral and district hospitals is not established. The equipment is very expensive and requires the presence of skilled personnel who may not be available. If the findings are equivocal or the operator is inexperienced the patient may need referral to a major centre for the transoesophageal echocardiography to be repeated, or for confirmation by aortography. Some may question the cost:benefit ratio, but we feel that the availability of transoesophageal echocardiography in our hospital may have saved these teenagers'. lives. There are currently enough useful applications of transoesophageal echocardiography for the assessment of patients intraoperatively, in intensive care, in the trauma unit, and the cardiology clinic that we feel-where the necessary experience is available-the cost of the equipment is justified.

In summary, this is the first published report of two occupants of the same vehicle sustaining aortic disruption. Both aortic disruptions were correctly diagnosed in a district general hospital using transoesophageal echocardiography before transfer to a regional cardiothoracic centre. These cases emphasise the need for a high degree of suspicion of aortic injury and the value of transoesophageal echocardiography in this setting.

The authors thank Mrs C Fuller and Mr S Large for their help with these cases.

1 Strassman G. Traumatic rupture of the aorta. Am Heart $\mathcal{f}$ 1947;33:508-15.

2 Brooks SW, Young JC, Cmolik B, Schina M, Dianzumba S, Townsend RN, et al. The Use of transesophageal echocardiography in the evaluation of chest trauma. $\mathcal{F}$ Trauma 1992;36:761-5.

3 Parmley LF, Mattingly TW, Manion WC, Jahnke EJ. Nonpenetrating traumatic injury of the aorta. Circulation 1958;17:1086-101.

4 Bennett DE, Cherry JK. The natural history of traumatic aneurysms of the aorta. Surgery 1967;61:516-23.

5 Gundry SR, Burney RE, Mackenzie JR, Jafri SZ, Shirazi K, Cho KJ. Traumatic pseudoaneurysms of the thoracic aorta. Anatomic and radiologic correlation. Arch Surg 1984;119:1055-60.

6 Fernandez LG, Lain KY, Messersmith RN, Jairam S, Gordon RT, Shah MR, et al. Transesophageal echocardiography for diagnosing aortic injury: a case report and Ro 36:77-80.

7 Smith MD, Cassidy M, Souther S, Morris EJ, Sapin PM, Johnson SB, et al. Transesophageal echocardiography in the diagnosis of traumatic rupture of the aorta. $N$ Engl f the diagnosis of trauma $1995 ; 332: 356-62$.

8 Raptopoulos V, Sheiman R, Phillips D, Davidoff A, Silva WE. Traumatic aortic tear: screening with chest CT WE. Traumatic aortic tea
Radiology 1992;182:667-3.

9 American College of Surgeons' Committee on Trauma: Advanced trauma life support. Chicago: American College of Surgeons, 1993:120.

10 Kàralis DG, Victor MF, Davis GA, McAllister MPJ, Covalesky VA, Ross J, et al. The role of echocardiography in blunt chest trauma: a transthoracic and transesophageal echocardiographic study. $\mathcal{F}$ Trauma $1994 ; 36$ : 53-8.

11 Daniel WG, Erbel R, Kasper W, Visser CA, Engberding R, Sutherland GR, et al. Safety of transesophageal echocarCirculation 1991;83:817-21.

12 Sparks MB, Burchard KW, Marrin CA, Bean $\mathrm{CH}$, Nugent WC, Plehn JF. Transoesophageal echocardiography. Preliminary results in patients with traumatic aortic rupPreliminary results in patients with
ture. Arch Surg 1991;126:711-13.

13 Unsworth-White MJ, Buckenham T, Treasure T. Traumatic rupture of the thoracic aorta: computed tomogramatic rupture of the thoracic aorta: computed tomogra-
phy may be a dangerous waste of time. Ann $R$ Coll Surg phy may be a dange

14 Banning AP, Ruttley MST, Musumeci F, Fraser AG Acute dissection of the thoracic aorta: transoesophageal Acute dissection of the thoracic aorta: transoesophageal
echocardiography is the investigation of choice. $B M 9$ 1995;310:72-3. 